\title{
CELLULAR MAPS BETWEEN POLYHEDRA
}

BY

JAMES P. HENDERSON

\begin{abstract}
A compact subset $X$ of a polyhedron $P$ is cellular in $P$ if there is a pseudoisotopy of $P$ shrinking precisely $X$ to a point. A proper surjection $f: P \rightarrow Q$ is cellular if each point inverse of $f$ is cellular in $P$. We give certain conditions under which cellular maps between polyhedra are approximable by homeomorphisms. An example of a cellular map which is not approximable by homeomorphisms is also given.
\end{abstract}

Introduction. The idea of generalizing the concept of cellularity to polyhedra was put forth by J. W. Cannon in [4]. He defined a compact subset $X$ of a polyhedron $P$ to be cellular in $P$ if there is a pseudoisotopy of $P$ shrinking precisely $X$ to a point, and a proper surjection $f: P \rightarrow Q$ between polyhedra to be a cellular map if for each $y \in Q$, the set $f^{-1}(y)$ is cellular in $P$. Cannon conjectured that if one could show that a cellular map $f: P \rightarrow Q$, where $P$ oi $Q$ is an $n$-manifold, $n \neq 4$, could be approximated by homeomorphisms, then one might be able to prove more general results involving more complicated polyhedra.

One of the basic techniques involved in working with polyhedral cellularity is the intrinsic stratification of polyhedra. It should be pointed out that we will be using a topological stratification and not a piecewise-linear stratification, as used by Aiken [1].

The first result which dealt with cellular maps between polyhedra which were not necessarily manifolds was that of Handel [8]. He was able to show that if $f: P \rightarrow Q$ is a cellular map with $P$ and $Q$ having no 4-dimensional stratum and for each $y \in Q$, both $y$ and $f^{-1}(y)$ lie in the same dimensional stratum of $P$ and $Q$ respectively, then $f$ is approximable by homeomorphisms. (Note that in general, a cellular set need not be contained in a single stratum.)

In [9], we were able to prove that if $f: P \rightarrow Q$ is a cellular map with either $P$ or $Q$ a generalized $n$-manifold, $n \neq 4$, or an $n$-manifold with boundary, $n \neq 4,5$, then $f$ is approximable. These conditions on $P$ and $Q$ were highly restrictive in the sense that either $P$ or $Q$ was required to have at most two different strata.

Later, we were able to show that if either $P$ and $Q$ had dimension at most 3, any cellular map $f: P \rightarrow Q$ is approximable by homeomorphisms. Again, the hypotheses were very restrictive, but the result encouraged us to believe that perhaps all cellular maps were approximable, with the possible requirement that the polyhedra have no

Received by the editors January 26, 1981 and, in revised form, September 8, 1981.

1980 Mathematics Subject Classification. Primary 57Q55, 54B15; Secondary 57N60, 57N80.

Key words and phrases. Cellular map, polyhedron, approximation, stratification. 
four-dimensional stratum. This conjecture could be seen as a direct analogy to the results of Armentrout [3], Siebenmann [14], and Edwards [5] for cellular maps between $n$-manifolds, $n \neq 4$.

In this paper we give an approximation theorem for cellular maps between polyhedra for which the main restrictions come from homotopy conditions placed on the way the strata of the polyhedra fit together. As usual, we also require that the four-dimensional stratum of the range polyhedron be empty $[9,10]$. We also require that each stratum in the domain have some piecewise-linear manifold structure. This is done only to allow the use of piecewise-linear engulfing.

Finally, an example of a cellular map between polyhedra is given which is not approximable by homeomorphisms. Thus, Question 4.9 of [11] is answered affirmatively. This example was pointed out to us by R. J. Daverman and we wish to thank him for allowing us to present it in this paper. He and J. J. Walsh have been especially helpful in the preparation of this paper.

1. Definitions and background. A polyhedron $P$ is a subset of some Euclidean space $\mathbf{R}^{n}$ such that each point $b \in P$ has a neighborhood $N=b L$, the join of $b$ and a compact subset $L$ of $P$. Throughout, $P$ and $Q$ will denote polyhedra. A compact subset $X$ of $P$ is cellular in $P$ if there is a pseudoisotopy $H_{t}: P \rightarrow P$ such that $X$ is the only nondegenerate point preimage of $H_{1}$. A proper surjection $f: P \rightarrow Q$ is a cellular map if, for each $y \in Q, f^{-1}(y)$ is cellular in $P$.

The intrinsic dimension of a point $x$ in $P$, denoted $I(x, P)$, is given by $I(x, P)=$ $\max \left\{n \in \mathbf{Z} \mid\right.$ there is an open embedding $h: \mathbf{R}^{n} \times c L \rightarrow P$ with $L$ a compact polyhedron and $h(\{0\} \times c)=x\}$, where $\mathrm{cL}$ is the open cone on $L$. The intrinsic $n$-skeleton of $P$ is $P^{(n)}=\{x \in P \mid I(x, P) \leqslant n\}$, and the intrinsic n-stratum of $P$ is $P[n]=P^{(n)}-P^{(n-1)}$. Define the depth of the stratification, $D(P)$, to be given by $D(P)=\max \{i-j \mid P[i] \neq \varnothing$ and $P[j] \neq \varnothing\}$. It is crucial to note that $P^{n}$ is a closed subpolyhedron of $P$ and that $P[n]$ is a topological $n$-manifold. (See [9] for more details.)

We state here some of the results on cellular maps for later use.

THEOREM 1.1 [9]. The following are equivalent:

(1) $X$ is a cellular subset of $P$.

(2) The projection $\pi: P \rightarrow P / X$ is approximable by homeomorphisms.

(3) $X=\cap_{i=1}^{\infty} N_{i}$, where the $N_{i}$ 's are homeomorphic cellular neighborhoods with $\bar{N}_{i+1} \subset N_{i}$.

A cellular neighborhood is an open set in $P$ which is homeomorphic to $\mathbf{R}^{n} \times \mathrm{cL}$, the neighborhoods used to describe the intrinsic dimension of points in $P$.

THEOREM 1.2 [10]. Let $f: P \rightarrow Q$ be a cellular map with $Q[4]=\varnothing$. Given $a$ component $B$ of a stratum $Q[n]$, there is a component $A$ of $P[n]$ such that $f \mid \bar{A}: \bar{A} \rightarrow \bar{B}$ is a cellular map.

TheOREM 1.3 [10]. Let $f: P \rightarrow Q$ be a cellular map with $Q[4]=\varnothing$. Then $f_{j}=f \mid P^{(j)}$ : $P^{(j)} \rightarrow Q^{(j)}$ is a cellular map and $P[n] \neq \varnothing$ if and only if $Q[n] \neq \varnothing$. 
THEOREM $1.4[9,10]$. Let $f: P \rightarrow Q$ be a cellular map with

(1) either $\operatorname{dim} P \leqslant 3$ or $\operatorname{dim} Q \leqslant 3$ or

(2) either $P$ or $Q$ is a generalized $n$-manifold, $n \neq 4$. Then $f$ is approximable by homeomorphisms.

The last theorem to be given in this section is a consequence of Handel's proof of his approximation theorem [8] and the fact that $f_{l}: P[l] \rightarrow Q[l]$ is a cellular map.

THeOREM 1.5. Let $f: P \rightarrow Q$ be a cellular map with $Q[4]=\varnothing$. Then $f$ may be approximated by cellular maps $\tilde{f}$ such at $\tilde{f}_{l}$ is a homeomorphism.

2. An engulfing theorem. We present here an engulfing theorem which will be used later to construct homeomorphisms with compact support in a stratum. The theorem given is in a sense a generalization of Stallings engulfing [16], and it has a radial engulfing analog given by Ancel in his notes on engulfing [2]. We will not give a rigorous proof of the theorem, but a short intuitive sketch of how the proof is based on Stalling's proof.

THEOREM 2.1. Let $M^{n}$ be a piecewise-linear $n$-manifold, $n \geqslant 5$. Suppose that for an integer $r, 0 \leqslant r \leqslant n-3$, there exist open sets $\tilde{V}_{i}$ and $\tilde{W}_{i+1},-1 \leqslant i \leqslant r$, such that $\tilde{V}_{i+1} \subset \tilde{V}_{i}, \tilde{W}_{i+2} \subset \tilde{W}_{i+1} \subset \tilde{V}_{i}$, and each i-complex in $\tilde{V}_{i}$ may be homotoped into $\tilde{W}_{i}$ rel $\tilde{W}_{i+1}$ by a homotopy in $\tilde{V}_{i-1}$.

Then given a closed complex $J$ in $\tilde{V}_{r}$ with $\operatorname{dim} J \leqslant n-3$ and a subcomplex $L$ of $J$ in $\tilde{W}_{r+1}$ such that $\operatorname{cl}(J-L)$ is the polyhedron of a finite $r$-subcomplex $R$ of $J$, there is a ambient isotopy $h_{t}: M \rightarrow M$ and a compact subset $E$ of $\tilde{V}_{-1}$ such that $h_{1}\left(\tilde{W}_{0}\right) \supset K$ and $h_{t} \mid(M-E) \cup L=\mathrm{id}$.

SKeTCH OF PROOF. If $r=0$, then for each point $x \in \operatorname{cl}(J-L)$, we can find a path from $x$ to $\tilde{W}_{0}$ which lies in $\tilde{V}_{-1}$. These paths may be chosen to miss $J-x$ for each $x$, and to be pairwise disjoint. The open set $\tilde{W}_{0}$ may now be stretched out along each of these paths to cover $J$.

Assume the theorem true for integers less than $r$. By hypothesis, there is a homotopy $g_{t}: J \rightarrow \tilde{V}_{r-1}$ such that $g_{t} \mid L=$ id and $g_{1}(J) \subset \tilde{W}_{r}$. As in Stalling's proof, we may assume that $g_{t}$ is a "nice" homotopy and that the only obstruction to using the set $g(J \times I)$ as a guide in pulling $\tilde{W}_{0}$ out to cover $J=g(J \times\{0\})=g_{0}(J)$ is a singularity set of $g_{t}$ and its shadow. This shadow set will be of dimension less than $r$, and it will lie in $\tilde{V}_{r-1}$. Hence our inductive assumption will allow us to engulf the shadow of the singularity set with $\tilde{W}_{0}$ keeping fixed any part of the $r$-skeleton of $g(J \times I)$ already in $\tilde{W}_{0}$. Thus we may engulf one simplex of the homotopy at a times, possibly uncovering only points in the interior of $(n-2)$-simplices which were previously covered, until we have engulfed $g(J \times\{0\})=J$.

3. Extending homeomorphisms of a stratum. In order to be able to use the engulfing theorem, we must be able to extend an engulfing homeomorphism defined on a stratum to a homeomorphism of the entire polyhedron. In this section we show that a homeomorphism $h: P[n] \rightarrow P[n]$ that is isotopic to the identity with compact support may be extended to a homeomorphism $\tilde{h}: P \rightarrow P$ which is also isotopic to the identity with compact support. 
LEMMA 3.1. Let $h_{t}: \mathbf{R}^{n} \rightarrow \mathbf{R}^{n}$ be an isotopy with compact support. There is an isotopy $\tilde{h}_{t}: \mathbf{R}^{n} \times \mathrm{cL} \rightarrow \mathbf{R}^{n} \times \mathrm{cL}$ with compact support such that $\tilde{h}_{t} \mid \mathbf{R}^{n}=h_{t}$.

Proof. If we express the open cone $\mathrm{cL}$ as $(L \times[0, \infty)) /(L \times\{0\})$, each point in $\mathbf{R}^{\mathrm{n}} \times \mathrm{cL}$ has a representation $(y, s, x)$ where $y \in \mathbf{R}^{n}, s \in[0, \infty)$, and $x \in L$. Define $\tilde{h}_{t}$ by

$$
\tilde{h}_{t}(y, s, x)= \begin{cases}\left(h_{t-s}(y) s, x\right), & t \geqslant s, \\ \left(h_{0}(y), s, x\right), & t \leqslant s .\end{cases}
$$

The next proposition was noted by Haver (see [7]) as a consequence of handle straightening techniques of Edwards and Kirby [6].

Proposition 3.2 [7]. If $M^{n}$ is an n-manifold and $H: M^{n} \times I \rightarrow M^{n}$ is an ambient isotopy with compact support, then there is a finite collection of isotopies $H_{i}: M^{n} \times$ $[i / k,(i+1) / k] \rightarrow M^{n}, 0 \leqslant i \leqslant k-1$ such that

(1) each $H_{i}$ has compact support in the interior of an $n$-cell $B_{i} \subset M$,

(2) $H_{0}\left(M^{n} \times\{0\}\right)=\mathrm{id}$,

(3) $H_{i}\left(M^{n} \times\{(i+1) / k\}\right)=H_{i+1}\left(M^{n} \times\{(i+1) / k\}\right)$,

(4) $H_{k-1}\left|\left(M^{n} \times 1\right)=H\right|\left(M^{n} \times 1\right)$.

TheOREM 3.3. Let $H: P[n] \times[0,1] \rightarrow P[n]$ be an ambient isotopy with compact support $E$ in $P[n]$. Then given a neighborhood $U$ of $P[n]$ in $P$, there is an ambient isotopy $\tilde{H}: P \times[0,1] \rightarrow P$ such that

(1) $\tilde{H}$ has compact support in $U$,

(2) $\tilde{H}|(P[n] \times 1)=H|(P[n] \times 1)$.

Proof. We first apply Proposition 3.2 to obtain a finite sequence of isotopies $H_{i}$ : $P[n] \rightarrow P[n]$, each having compact support in an $n$-cell $B_{i}$ in $P[n]$ such that $H_{k-1}|(P[n] \times 1)=H|(P[n] \times 1)$.

Given an open subset $W$ of $P[n]$ which is homeomorphic to $\mathbf{R}^{n}$ and a neighborhood $U$ of $\bar{W}$, there is an open set $\tilde{W}$ in $P$ such that $\tilde{W} \cap P[n]=W, \tilde{W} \cong \mathbf{R}^{n} \times \mathrm{cL}$, and $\mathrm{cl} \tilde{W} \subset U\left[\mathbf{8 , 1 2}\right.$. Thus for each $B_{i}$, we can find an open set $U_{i}$ in $P$ such that $U_{i} \cong \mathbf{R}^{n} \times \mathrm{cL}, U_{i} \cap P[n]=$ int $B_{i}$, and $\bar{U}_{i} \subset U$. We now apply Lemma 3.1 to get an extension $\tilde{H}_{i}$ of $H_{i}$ to $P$ which has compact support in $U_{i}$. The desired isotopy $\tilde{H}$ is then the composition $\tilde{H}_{k-1} \cdots \tilde{H}_{1}: P \rightarrow P$.

4. The approximation theorem. Before stating the main result, we recall that if $X \subset Y, X$ is $i-L C C$ in $Y$ if for each $x \in X$ and neighborhood $U_{x}$ of $x$, there is a neighborhood $V_{x}$ of $x$ such that each map of an $i$-sphere into $V_{x}-X$ is null homotopic in $U_{x}-X$.

THEOREM 4.1. Let $P$ and $Q$ be polyhedra such that

(1) $Q[4]=\varnothing$,

(2) each $P[n]$ has a piecewise-linear triangulation,

(3) for each $n \geqslant 5, \overline{P[n]}-P[n]$ is $i-L C C$ in $\overline{P[n]}, i=12$,

(4) for each $n \geqslant 5, \overline{Q[n]}-Q[n]$ is 1-LCC in $\overline{Q[n]}$.

Then each cellular map $f: P \rightarrow Q$ is approximable by homeomorphisms. 
Proof. The proof will be by induction on $D(P)$, the depth of stratification of $P$. If $D(P)=0$, it follows from Theorem 1.3 that $n \neq 4$. Hence $f$ is approximable by homeomorphisms according to Theorem 1.4.

We therefore assume that $D(P) \geqslant 1$. It may also be assumed that $P[0]=\varnothing$ (Theorem 1.1 implies that any discrete collection of cellular sets may be shrunk out), and that $f_{l}: P[l] \rightarrow Q[l]$ is a homeomorphism by Theorem 1.5. The induction step will consist of showing that $f$ can be approximated by a cellular map $\tilde{f}: P \rightarrow Q$ such that $\tilde{f}$ is $1-1$ over $Q[l]$. We would then be able to apply the inductive hypothesis to approximate $\tilde{f} \mid P-P[l]$ by a homeomorphism which may be extended to $P[l]$ by $\tilde{f}_{l}$.

In order to complete the necessary step, it suffices to prove the following inductive statement and follow that with an application of the Bing shrinking criterion.

$I(j, k)$ : For each $k$-dimensional subpolyhedron $K$ of $P[l], \varepsilon: P \rightarrow(0,1)$, and neighborhood $U$ of $f^{-1}(f(K))$, there is a homeomorphism $h: P \rightarrow P$ such that

(1) $h \mid(P-U) \cup P[l]=\mathrm{id}$,

(2) for each $x \in K$, diam $h\left(f^{-1}(f(x))\right) \cap P^{(j)}<\varepsilon(x)$,

(3) for each $x \in P, d(f h(x), f(x))<\bar{\varepsilon}(x)$.

Note first that $I(l, k)$ is true for each $k$ with $h=$ id. Also, $I(j, 0)$ is true for each $j$ since $f^{-1}(f(K))$ will consist of a discrete collection of cellular subsets of $P$. Given such a discrete collection, $\varepsilon: P \rightarrow(0,1)$, and neighborhood $U$, one can find cellular neighborhoods $N_{x}, x \in K$, such that

(1) $N_{x} \supset f^{-1} f(x)$ for $x \in K$,

(2) $N_{x} \cap N_{y}=\varnothing$ for $x \neq y$,

(3) $\bar{N}_{x} \subset U$ for each $x$,

(4) $\operatorname{diam} f\left(N_{x}\right)<\min \left\{\varepsilon(z) \mid z \in N_{x}\right\}$.

The cone structure on each $N_{x}$ may now be employed to define a homeomorphism $h$ : $P \rightarrow P$ with the desired properties.

The proof of the inductive statement can now be completed by showing that the statements $I(j-1, k)$ and $I(j, k-1)$ imply $I(j, k)$.

Let $K, U$, and $\varepsilon: P \rightarrow(0,1)$ be given. Triangulate $K$ in such a way that for each $k$-simplex $\sigma$ in $K, \operatorname{diam} \sigma<\delta=\min \left\{\frac{1}{4} \in(x) \mid x \in \sigma\right\}$. It follows from $I(j, k-1)$ that we may assume that, for each $x \in \partial \sigma$, diam $f_{j}^{-1} f_{j}(x)<\delta$ and from $I(j-1, k)$ that, for each $x \in \sigma$, diam $f_{j-1}^{-1} f_{j-1}(x)<\delta$. Let $N_{\sigma} \cong \mathbf{R}^{l} \times \mathrm{cL}$ be a neighborhood of $f_{j}$ (int $\left.\sigma\right)$ in $Q^{(j)}$ such that

(1) $N_{\sigma} \subset f_{j}\left(U \cap P^{(j)}\right)$,

(2) $\operatorname{diam} N_{\sigma}<\delta$,

(3) $f_{j}^{-1}\left(N_{\sigma}\right) \cap(K-\sigma)=\varnothing$,

(4) if we identify $N_{\sigma}$ with $\mathbf{R}^{l} \times \mathrm{cL}$, there is an $l$-cell $B$ in $\mathbf{R}^{l}$ such that, for $y \in \mathbf{R}^{l}-$ int $B, \operatorname{diam}\left(f_{j}^{-1}(y \times \mathrm{cL})\right)<\delta / 4$.

Now $U_{\sigma}=f_{j}^{-1}\left(N_{\sigma}\right)$ is a neighborhood of $f_{j}^{-1} f_{j}($ int $\sigma)$ which lies in $U$.

Assume that $l<j \leqslant 3$. We may use Theorem 1.4 to approximate $f_{j} \mid U_{\sigma}: U_{\sigma} \rightarrow N_{\sigma}$ by a homeomorphism $h_{\sigma}$ such that, for $y \in \mathbf{R}^{l}-$ int $B$, diam $h_{\sigma}^{-1}(y \times \mathrm{cL})<\delta / 4$. Let $A=h_{\sigma}^{-1}(B)$.

In [10], the possible structures of cellular neighborhoods $\mathbf{R}^{l} \times \mathrm{cL}$ of dimension at most three were described in detail. We will not repeat that analysis here, but we will 
describe the various situations and refer the reader to that paper for further details.

In the case where $l=1$ and $j=2, \mathbf{R}^{1} \times \mathrm{cL} \cong U_{\sigma}$ is homeomorphic to a finite number of copies of $\mathbf{R}_{+}^{2} \cong \mathbf{R}^{1} \times[0, \infty)$ identified along their common $\mathbf{R}^{1}$ boundary. In this situation we can find an isotopy $h_{t}$ with compact support in each $A \times[0, \infty)$ which shrinks each $f_{2}^{-1} f_{2}(x)$ to have diameter less than $\varepsilon(x)$ for each $x \in U_{\sigma} \cap P[1]$ and is the identity on $\partial A \times[0, \infty) \cup A \times\{0\}$. The homeomorphism $h_{1}$ can be extended to $\tilde{H}_{\sigma}: P \rightarrow P$ with support in $U$ in such a way that for $x \in K-f_{2}^{-1} f_{2}$ (int $\sigma$ ), $f^{-1} f(x)$ is not moved. Piecing together these homeomorphisms, one for each $k$-simplex $\sigma$ in $K$, yields the desired homeomorphisms. A similar argument holds for $l=2$ and $j=3$.

In the case where $l=1$ and $j=3$, we have by assumption that, for each $x \in \sigma$, $f_{2}^{-1} f_{2}(x)<\delta$. Thus we need only consider the 3 -dimensional stratum of $\mathbf{R}^{1} \times \mathrm{cL}$. It was shown in [10] that the 3-stratum can be dealt with by considering the set $\mathbf{R}^{1} \times c\left(I^{1}\right)$ or $\mathbf{R}^{1} \times c\left(S^{1}\right)$, with each $\mathbf{R}^{\mathbf{l}} \times c$ corresponding to the $\mathbf{R}^{\mathbf{l}} \times c$ in $\mathbf{R}^{\mathbf{l}} \times \mathrm{cL}$.

For $\mathbf{R}^{1} \times c\left(S^{1}\right)$, one may proceed as in the above cases to find an isotopy with compact support in (int $\left.A \times c S^{1}\right)$ - (int $A \times c$ ) which shrinks $f_{3}^{-1} f_{3}(x)$ to have diameter less than $\varepsilon(x)$. Again, we extend the final stage of the isotopy to all of $P$.

When considering $\mathbf{R}^{1} \times c\left(I^{1}\right)$, we also identify a 1-cell $D$ in int $I^{1}$ such that, for each $x \in$ int $A$ such that diam $f_{3}^{-1} f_{3}(x)>\varepsilon(x), f_{3}^{-1} f_{3}(x) \subset$ int $A \times c($ int $D)$. There is an isotopy of $\mathbf{R}^{1} \times c\left(I^{1}\right)$ with compact support in (int $\left.A\right) \times c($ int $D)-($ int $A) \times c$ which shrinks each $f_{3}^{-1} f_{3}(x)$ to small size. The final stage can once more be extended to $P$ yielding the desired result.

The hypothesis that $Q[4]=\varnothing$ and Theorem 1.3 together imply that $P[4]=\varnothing$. Therefore the statement $I(3, k)$ implies $I(4, k)$.

We now consider $I(j, k)$ for $j \geqslant 5$. Let $N_{\sigma}$ and $U_{\sigma}$ be as above, and let $U_{j}=U_{\sigma} \cap$ $P[j]$. We can assume that $U_{j}$ has only one component; otherwise we would work in one component of $U_{j}$ at a time. In order to shrink each $f_{j}^{-1} f_{j}(x)$ which intersects $U_{j}$, $x \in$ int $\sigma$, we will need two engulfing lemmas. The lemmas will be stated, the proof of $I(j, k)$ completed, and then the proofs of the two lemmas given.

Let $X=f^{-1} f(\sigma) \cap U_{j}$ and $C=\left[f^{-1} f(\sigma-\right.$ int $\left.A)\right] \cap U_{j}$.

Lemma 1. There exist an open set $V$ in $U_{j}$ with $X \subset V$ and open sets $W$ and $N$ in $U_{j}$ for which

(1) $\bar{W} \subset N \subset \bar{N} \subset V$ (closures taken in $V)$,

(2) $N \subset N_{\delta}(\sigma ; P) \cap U_{j}$,

(3) $C \subset W$,

such that for each polyhedron $J$ in $V$ of dimension at most $n-3$ with subpolyhedron $L \subset N$ and $\overline{J-L}$ compact, there is a homeomorphism $h_{1}: U_{j} \rightarrow U_{j}$ which is isotopic to the identity with compact support in $U_{j}-\mathrm{cl} W$ such that $h_{1}(N) \supset J$.

LEMMA 2. Let $V$ be the open set given in Lemma 1. For each polyhedron $J$ in $V$ with $\operatorname{dim} J \leqslant 2$ and subpolyhedron $L$ of $J$ for which $L \subset V-X$ and $\overline{J-L}$ is compact, there is a homeomorphism $h_{2}: V \rightarrow V$ isotopic to the identity with compact support in $V$ such that $h_{2}(V-X) \supset J$. 
We now complete the proof of $I(j, k)$. Let $S$ be a triangulation of $V$, and let $R$ be the 2-skeleton of $\operatorname{st}(V-W, S)$. An application of Lemma 2 yields a homeomorphism $h_{2}: V \rightarrow V$ such that $h_{2}(V-X) \supset R$ and $h_{2}$ is isotopic to the identity with support in a compact subset $E$ of $V$. Define $J^{*}=\{\tau \in S \mid \tau \subset \bar{W} \cup(V-(E \cup X))\}$ and let $J$ be the dual codimension-three polyhedron in $V$. There is a homeomorphism $h_{1}: U_{j} \rightarrow U_{j}$ provided by Lemma 1 such that $h_{1}(N) \supset J$ and $h_{1}$ have compact support in $U_{j}-\operatorname{cl} W$. We extend $h_{1}$ by the identity to $U_{j}$ and then use the join structure between $R$ and $J$ in $V$ to obtain a homeomorphism $h_{3}: U_{j} \rightarrow U_{j}$ such that $h_{1}(N) \cup h_{3} h_{2}\left(U_{j}-X\right)=U_{j}$. The homeomorphisms $h_{\sigma}=h_{1}^{-1} h_{3} h_{2}$ may be extended to all of $P$ in such a way that no points of $f^{-1} f(K-$ int $\sigma) \cup P-U$ are moved, and $h_{\sigma}(X) \subset N$. The proof of $I(j, k)$ is completed by composing homeomorphisms defined as above for each $k$-simplex $\sigma$ in $K$.

PROOF OF LEMMA 1. The proof will consist of constructing neighborhoods $V_{i}$ of $f_{j}^{-1} f_{j}$ (int $\left.\sigma\right)$ in $U_{\sigma} \cap P^{(j)}$, and neighborhoods $W_{i}$ of int $\sigma$ in $U_{\sigma} \cap P^{(j)}$ such that $V_{i} \subset V_{i-1}, W_{i+1} \subset W_{i} \subset N_{\delta}$ (int $\sigma ; P$ ), $W_{i+1} \subset V_{i}$, and a neighborhood $W$ of int $\sigma \cup$ $f_{j}^{-1} f_{j}(\sigma-$ int $A)$ with $W \subset W_{i}$ such that $V_{i}-\bar{W}$ may be homotoped stratum preserving into $W_{i}-\bar{W}$ rel $W_{i+1}$ in $V_{i-1}-\bar{W}$ for $0 \leqslant i \leqslant j-3$. We will then apply Theorem 2.1 to the manifold $U_{j}-\bar{W}$ with $\tilde{V}_{i}=\left(V_{i}-\bar{W}\right) \cap P[j]$ and $\tilde{W}_{i}=\left(W_{i}-\right.$ $\bar{W}) \cap P[j]$. The desired set $V$ is $\tilde{V}_{j-3}$.

The homotopies and neighborhoods will be constructed in the same manner as those of Proposition 2.2 of [10]. Choose a neighborhood $R$ of int $\sigma$ in $U_{\sigma} \cap P^{(j)}$ such that

(1) for each $x \in$ int $\sigma-$ int $A, f_{j}^{-1} f_{j}(x) \subset R$,

(2) $R \subset N_{\delta}(\sigma ; P)$.

Cover $f_{j}^{-1} f_{j}$ (int $\sigma$ ) with a locally finite collection of saturated open sets $\left\{N_{\alpha}^{k}\right\}$ such that

(1) $\cup N_{\alpha}^{k} \subset U_{\sigma}$,

(2) for each $N_{\alpha}^{k}$, there is a cellular neighborhood $C_{\alpha}^{k}$ of the form $\mathbf{R}^{l} \times \mathrm{cL}$ such that $\bar{N}_{\alpha}^{k} \subset C_{\alpha}^{k} \subset \bar{C}_{\alpha}^{k} \subset U_{\sigma}$,

(3) for each $C_{\alpha}^{k}$, if $C_{\alpha}^{k} \cap\left(\right.$ int $\sigma-$ int $A$ ) $=\varnothing$, then $\bar{C}_{\alpha}^{k} \subset R$.

Let $T_{k}$ be a triangulation of int $\sigma$ such that bd $A$ is a subcomplex and that, for each $\tau \in T_{k}, f_{j}^{-1} f_{j}(\tau)$ lies in some $U_{\alpha}^{k}$. Then for each $(k-1)$-simplex $\gamma$ in the $(k-1)$-skeleton $T_{k}^{k-1}$ of $T_{k}$, cover $f_{j}^{-1} f_{j}(\gamma)$ by a finite number of saturated open sets $\left\{U_{\beta}^{k-1}\right\}$ such that for each $U_{\beta}^{k-1}$ there is a cellular neighborhood $C_{\beta}^{k-1} \cong \mathbf{R}^{l} \times \mathrm{cL}$ for which $f_{j}^{-1} f_{j}(\gamma) \subset U_{\alpha}^{k}$ implies that $\bar{U}_{\beta}^{k-1} \subset C_{\beta}^{k-1} \subset \bar{C}_{\beta}^{k-1} \subset U_{\alpha}^{k} . T_{k-1}$ will then be a subdivision of $T_{k}^{k-1}$ such that, for each simplex $\tau \in T_{k-1}, f_{j}^{-1} f_{j}(\tau) \subset U_{\beta}^{k-1}$ for some $\beta$. Similarly define $T_{n-1},\left\{U_{\beta}^{n-1}\right\}$, and $\left\{C_{\beta}^{n-1}\right\}$ given $T_{n},\left\{U_{\alpha}^{n}\right\}$, and $\left\{C_{\alpha}^{n}\right\}$. We also require that $\left\{C_{\beta}^{0}\right\}$ be a pairwise disjoint collection of cellular neighborhoods.

We will define $V_{-1}=U_{j}$, and now describe $V_{0}$ and $W_{0}$. Let $A_{0}=\cup\left\{U_{\beta}^{0}\right\}$, and for each 1-simplex $\tau \in T_{1}$, define $A_{\tau}$ to be a saturated open set containing $f_{j}^{-1} f_{j}\left(\tau-A_{0}\right)$ which lies in some $U_{\alpha}^{1}$. We also require that if $\tau$ and $\gamma$ are different 1-simplices in $T_{1}$, then $\overline{A_{\tau}} \cap \overline{A_{\gamma}}=\varnothing$. Define $A_{1}=\bigcup\left\{A_{\tau} \mid \tau\right.$ is a simplex in $\left.T_{1}\right\}$. Similarly construct $A_{n}, 1 \leqslant n \leqslant k$, and let $V_{0}=\cup_{n=0}^{k} A_{n}$. 
The desired homotopy will first pull $A_{0}$ into $R$ in such a way that those neighborhoods $A_{\tau}$ of 0 -simplices $\tau$ which lie in int $\sigma-$ int $A$ are fixed, while the other neighborhoods used to define $A_{0}$ are pulled close to $P[l]$ using the cone structure on the $C_{\beta}^{0}$ 's. This may be done so that the subsequent homotopies will not move the image of $A_{0}$, and so that no points are moved in a neighborhood of $P[l] \cup f_{j}^{-1} f_{j}$ (int $\sigma-$ int $D_{1}$ ), where $D_{1}$ is a $k$-cell contained in $A$. Let $H_{t}^{0}: V_{0} \rightarrow V_{-1}$ be this homotopy. In the same manner, construct homotopies $H_{t}^{n}: H_{1}^{n-1} \cdots H_{1}^{0}\left(V_{0}\right)$ $\rightarrow V_{-1}$ such that $H_{t}^{n} \mid H_{1}^{n-1} \cdots H_{1}^{0}\left(\cup_{j<n} A_{j}\right)=\mathrm{id}, H_{1}^{n}\left(\cup_{j=0}^{n} A_{j}\right) \subset R$, and $H_{t}^{n}$ moves no points in a neighborhood of $P[l] \cup f_{j}^{-1} f_{j}$ (int $\sigma-$ int $D_{n}$ ), where $D_{n}$ is a $k$-cell contained in $D_{n-1}$. The desired homotopy $G$ is then $G=H^{k} H^{k-1} \cdots H^{0}: V_{0} \rightarrow V_{-1}$. Let $W_{0}$ be a neighborhood of $G_{1}\left(V_{0}\right)$ which lies in $R$. Note also that there is a neighborhood $W_{1}^{*}$ of $f_{j}^{-1} f_{j}\left(D_{k}\right) \cup P[l]$ which lies in $G_{1}\left(V_{0}\right)$ on which $G_{t}$ is the identity. We now repeat the above construction with $V_{-1}$ replaced by $V_{0}, W_{1}^{*}$ replacing $R$, and identify $V_{1} \subset V_{0}, W_{1} \subset W_{0}$, and $W_{2}^{*} \subset W_{1}$. The sets $V_{n}, W_{n}$, and $W_{n+1}^{*}$ are thus defined inductively. Finally, $W$ is a neighborhood of $P[l] \cup$ $f_{j}^{-1} f_{j}$ (int $\sigma-$ int $A$ ) whose closure lies in $W_{j-1}^{*}$.

We note here for later use that we could have modified this procedure to describe a neighborhood $D$ of $f_{j}^{-1} f_{j}$ (int $\sigma$ ) in $V_{j-3}$ and a homotopy $h_{t}: D \rightarrow V_{j-3}$ such that for each $x \in D$ there is $s_{x} \in[0,1)$ for which $I\left(h_{t}(x), P\right)=I(x, P)$ for $t<s_{x}$ and $I\left(h_{t}(x), P\right)=l$ for $t \geqslant s_{x}$. The changes required would be to cover all of $f_{j}^{-1} f_{j}($ int $\sigma)$ and have the homotopies use the cellular neighborhoods to pull each $A_{i}$ into $P[l]$ keeping $A_{i} \cap P[l]$ fixed.

Proof of Lemma 2. We wish to apply Stallings' engulfing theorem [16] to the manifold $V$. Thus we must show that $(V, V-X)$ is 2-connected.

Let $x$ be a point in $X$. Since $V_{j-3} \supset f_{j}^{-1} f_{j}$ (int $\left.\sigma\right)$, there is a cellular neighborhood $U_{x}$ containing $f_{j}^{-1} f_{j}(x)$ with $U_{x} \subset V_{j-3}$. Since $f_{j}\left(U_{x}\right) \not \subset Q^{(j-1)}$ by Theorem 1.2, there is a point $y \in\left(U_{x}-f_{j}^{-1} f_{j}\right.$ (int $\left.\left.\sigma\right)\right) \cap P[j]$. It now follows that there is a path from $x$ to $y$ in $U_{x} \cap P[j]$ and $(V, V-X)$ is 0 -connected.

Suppose that $g:\left(B^{1}, \partial B^{1}\right) \rightarrow(V, V-X)$ is given, where $B^{1}$ is a 1-cell. As was noted before, there is a neighborhood $D$ of $f_{j}^{-1} f_{j}$ (int $\left.\sigma\right)$ in $V_{j-3}$ and a homotopy $h_{t}$ : $D \rightarrow V_{j-3}$ such that, for each $x \in D, I\left(h_{t}(x), P\right)=I(x, P)$ for $t<s_{x}$ and $I\left(h_{t}(x), P\right)=l$ for $t>s_{x}$. Let $\tau_{1}, \tau_{2}, \ldots, \tau_{n}$ be disjoint arcs in $B^{1}$ such that

(1) $g\left(\tau_{i}\right) \cap X \subset g\left(\right.$ int $\left.\tau_{i}\right)$,

(2) $g\left(B^{1}\right) \cap X \subset \cup_{i=1}^{n} g\left(\right.$ int $\left.\tau_{i}\right) \subset \cup_{i=1}^{n} g\left(\tau_{i}\right) \subset D \cap P[j]$.

Given a component $U$ of $D \cap P[j], U-f_{j}^{-1} f_{j}(\sigma)$ is path connected (see Proposition 2.3 of $[\mathbf{1 0}])$. Hence we may connect the points of $g\left(\partial \tau_{i}\right)$ by a path $\alpha_{i}$ : $\tau_{i} \rightarrow(D \cap P[j])-X$. Since $D$ may be homotoped to $D \cap P[l]$ in a stratum respecting manner, the singular loop $\alpha_{i}\left(\tau_{i}\right) \cup g\left(\tau_{i}\right)$ bounds a singular disk in $V \cup(D \cap$ $P[l])$. Since $V_{j-3} \cap P[l]$ is homeomorphic to $\mathbf{R}^{l}$ and hence has a product neighborhood $\mathbf{R}^{l} \times \mathrm{cL}$ in $V_{j-3}$, we can assume that the singular disk intersects $P[l]$ in a single point in $V_{j-3}$. That point may then be eliminated by the 1-LCC hypothesis in $P$. Now $\alpha_{i}$ is homotopic to $g \mid \tau_{i}$ in $V$, and it follows that $(V, V-X)$ is l-connected.

It remains to be shown that $(V, V-X)$ is 2-connected. If $g:\left(B^{2}, \partial B^{2}\right) \rightarrow(V, V-$ $X)$ is given, choose disjoint disks-with-holes $B_{1}, B_{2}, \ldots, B_{n}$ such that 
(1) $g\left(B_{i}\right) \cap X \subset g\left(\right.$ int $\left.B_{i}\right)$,

(2) $g\left(B^{2}\right) \cap X \subset \cup_{i=1}^{n} g\left(\right.$ int $\left.B_{i}\right)$,

(3) $g\left(B_{i}\right) \subset D \cap P[j]$,

where $D$ is the neighborhood described above.

We first want to approximate $f g \mid B_{i}: B_{i} \rightarrow N_{\sigma}$ by a map $k_{i}: B_{i} \rightarrow N_{\sigma}$ such that $k_{i}\left|\partial B_{i}=f g\right| \partial B_{i}$ and $k_{i}$ (int $\left.B_{i}\right) \subset N_{\sigma} \cap Q[j] \cap f_{j}(D)$. For each point $y \in f g\left(B_{i}\right) \cap$ $Q[k]$, let $N_{y}$ be a neighborhood of $y$ homeomorphic to $\mathbf{R}^{k} \times \mathrm{cL}_{k}$ which lies in $f_{j}(D)$. Cover $f g\left(B_{i}\right)$ by finitely many such neighborhoods $N_{1}, \ldots, N_{m}$ and let $T$ be a triangulation of int $B_{i}$ such that, for each simplex $\tau$ of $T$, diam $\tau<d\left(\tau, \partial B_{i}\right)$ and each $f g(\tau)$ lies in some $N_{s}$. For each point $y$ in the 0 -skeleton of $T$ with $f g(y) \in Q[k]$, we will approximate $f g(y)$ by a point in the component of $N_{y} \cap Q[j]$ which corresponds to the component of $f_{j}^{-1}\left(N_{y}\right) \cap P[j]$ containing $g(y)$. (See Theorem 1.2.) This is done so that the new map $k_{i}$ defined on the 0 -skeleton of $T$ sends each point into $Q[j] \cap N_{\sigma}$ so close to its image under $f g$ that, for each 1-simplex $\tau$ with $f g(\tau) \subset N_{s}, k_{i}(\partial \tau)$ again lies in $N_{s}$. We may connect the points $k_{i}(\partial \tau)$ in $N_{s} \cap Q[j]$ by an arc whose diameter is less than twice the diameter of $f g(\tau)$. This arc will then be $k_{i}(\tau)$. Now for each 2-simplex $\gamma$ in $T, k_{i}(\partial \gamma)$ is already defined, and lies in some $N_{s} \cap Q[j]$. This singular 1-sphere bounds a singular 2-cell in $\left(N_{s} \cap Q[j]\right) \cup\left(N_{s} \cap\right.$ $Q[k])$, when $N_{s} \cong \mathbf{R}^{k} \times \mathrm{cL}_{k}$. Again we may assume that this singular 2-cell intersects $Q[k]$ in a single point and use the 1-LCC condition on $Q$ to cut this singular 2-cell off in $Q[j]$. We now have a map $k_{i}$ : int $B_{i} \rightarrow Q[j] \cap f(D)$ which may be extended to $\partial B_{i}$ by the map $f g$. Thus if we define $k: B^{2} \rightarrow N_{\sigma}$ to be $k_{i}$ on $B_{i}$ and $f g$ on $B^{2}-\cup_{i=1}^{n} B_{i}$, we have a map of $B^{2}$ into $N_{\sigma}-f_{j}(\sigma)$.

An approximate lift $\tilde{k}: B^{2} \rightarrow V-X$ of the map $k: B^{2} \rightarrow N_{\sigma}-f_{j}(\sigma)$ is constructed so that, for each $i, \tilde{k}\left(B_{i}\right) \subset(D \cap P[j])-X$ and $\tilde{k}\left|\partial B_{i}=g\right| \partial B_{i}$. Cover $k\left(B_{i}\right)$ with cellular neighborhoods to get open covers $V_{0}=\left\{V_{01}, V_{02}, \ldots, V_{0 n}\right\}, V_{1}=$ $\left\{V_{1,1}, \ldots, V_{1, m}\right\}$, and $V_{2}=\left\{V_{2,1}, \ldots, V_{2, p}\right\}$ of $k\left(B_{i}\right)$ such that

(1) $\operatorname{st}^{2}\left(k(y), V_{r}\right) \subset V_{r+1, s}$ for some $s$, where $y \in B_{i}$,

(2) $f_{j}^{-1}\left(V_{2, k}\right)$ is contained in a cellular neighborhood in $D-f_{j}^{-1} f_{j}(\sigma), 1 \leqslant k \leqslant p$.

Triangulate $B_{i}$ so that for each simplex $\tau$ of the triangulation, $k(\tau) \subset V_{0, s}$ for some $s$. Define the approximate lift on the 0 -skeleton so that, for each point $y \in \partial B_{i}$, $\tilde{k}(y)=g(y)$. Given a 2-simplex $\gamma$ of the triangulation and neighborhood $V_{0, s}$ containing $k(\gamma)$, we also require that the vertices of $\gamma$ be mapped by $\tilde{k}$ into the same component of $f_{j}^{-1}\left(V_{0, s}\right) \cap P[j]$. Thus for each 1-simplex $\tau$ of the triangulation, define $\tilde{k}(\tau)$ to be a path between the points of $\tilde{k}(\partial \tau)$ which lies in $f_{j}^{-1}\left(V_{1, r}\right) \cap P[j]$, where $V_{1, r} \supset k(\tau)$. Again we require that, for $\tau \subset \partial B_{i}, \tilde{k}(\tau)=g(\tau)$.

For each 2-simplex $\gamma$ in the triangulation, we have $\tilde{k}(\partial \gamma) \subset N_{\gamma} \cap P[j]$, where $N_{\gamma}$ is a cellular neighborhood lying in $D$ and containing $f_{j}^{-1}\left(V_{2, s}\right) \supset f_{j}^{-1}(k(\gamma))$. Hence $\tilde{k}(\partial \gamma)$ bounds a singular 2-cell in $\left(N_{\gamma} \cap P[j]\right) \cup\left(N_{\gamma} \cap P[n]\right)$ where $N_{\gamma} \cong \mathbf{R}^{n} \times \mathrm{cL}_{n}$. We again take this singular 2-cell so that it intersects $P[n]$ in a single point, and eliminate that point using the 1-LCC hypothesis on $P$. We now have a map $\tilde{k}$ : $B_{i} \rightarrow(D \cap P[j])-X$ which agrees with $g$ on $\partial B_{i}$. Because $g\left(B_{i}\right) \cup \tilde{k}\left(B_{i}\right) \subset D$, $g\left(B_{i}\right)$ is homotopic to $\tilde{k}\left(B_{i}\right)$ rel $\partial B_{i}$ in $V \cup(D \cap P[l])$. As before, we can make the homotopy intersect $P[l]$ in a single point and then use the 2-LCC condition on $P$ to cut the homotopy off of $P[l]$ in $P[j]$. 
Thus for each $i$, we have a map $\tilde{k} \mid B_{i}: B_{i} \rightarrow V-X$ such that $\tilde{k}\left|\partial B_{i}=g\right| \partial B_{i}$ and $\tilde{k} \mid B_{i}$ is homotopic to $g \mid B_{i}$ rel $\partial B_{i}$ in $V$. If we extend $\tilde{k}$ to $B^{2}-\cup_{i=1}^{n} B_{i}$ by the map $g$, we then have a map $\tilde{k}: B^{2} \rightarrow V-X$ which is homotopic to $g$ rel $\partial B_{i}$ in $V$.

5. Examples. Recently, R. J. Daverman pointed out the following example of a cellular map between polyhedra which is not approximable by homeomorphisms.

Let $W$ be a contractible $(n+1)$-manifold whose boundary is a nonsimply connected homology $n$-sphere $H^{n}, n \geqslant 4$, and choose $w_{0} \in W$. The polyhedron $P$ is then given by

$$
P=\left(W \times S^{1}\right) \underset{w_{0} \times S^{1}}{\cup}\left(W \times S^{1}\right),
$$

two copies of $W \times S^{1}$ identified along the circle $w_{0} \times S^{1}$ by the identity map. Choose $\tilde{W}$ to be a submanifold of $W$ containing $w_{0}$ such that $W-$ int $\tilde{W}=H \times[0,1]$. The polyhedron $Q$ is defined by

$$
Q=\left(\mathrm{cH} \times S^{1}\right) \underset{c \times S^{1}}{\cup}\left(\mathrm{cH} \times S^{1}\right),
$$

with $\mathrm{cH}$ being the standard cone on the homology sphere $H$. The map to be considered is $f: P \rightarrow Q$ which takes each $(W \times y) \cup_{w_{0} \times y}(W \times y)$ onto $(\mathrm{cH} \times y)$ $\cup_{c \times y}(\mathrm{cH} \times y)$ by sending $(\tilde{W} \times y) \cup_{w_{0} \times y}(\tilde{W} \times y)$ to the point $c \times y$.

We first note that $\tilde{W}$ is a cell-like subset of $W$, and hence that $\tilde{W} \times y$ is a cellular subset of $W \times S^{1}$. Since $w_{0} \times S^{1}$ is a tame 1-sphere in $W \times S^{1}$, given a neighborhood $U$ of $\tilde{W} \times y$ in $W \times S^{1}$, there is an open neighborhood $V$ of $\tilde{W} \times y$ in $W \times S^{1}$ such that $\bar{V} \subset U$ and a homeomorphism $h: V \rightarrow \mathbf{R}^{n+2}$ which takes $\left(w_{0} \times S^{1}\right) \cap V$ onto $\mathbf{R}^{1} \times\{0\} \subset \mathbf{R}^{n+2}$. It now follows from Theorem 1.1 that $\tilde{W} \times y \cup_{w_{0} \times y} \tilde{W} \times y$ is cellular in $P$.

In order to show that $f$ is not approximable by homeomorphisms, it suffices to show that $P[1]=w_{0} \times S^{1}$ is 1-LCC in $P$, but $Q[1]=c \times S^{1}$ is not 1-LCC in $Q$.

Given a point $x \in w_{0} \times S^{1}$ and a neighborhood $U$ of $x$ in $P$, there is a neighborhood $V$ of $x$ in $P$ such that $x \in V \subset U$ and $V \cong\left(W \times \mathbf{R}^{1}\right) \cup_{w_{0} \times \mathbf{R}^{1}}\left(W \times \mathbf{R}^{1}\right)$. Each loop in $V-P[1]$ can be contracted in $V$ missing $w_{0} \times \mathbf{R}^{1}$ since $w_{0} \times \mathbf{R}^{1}$ is tame in each of the contractible manifolds $W \times \mathbf{R}^{1}$.

On the other hand, given a neighborhood $U$ of a point $y \in Q[1] \cong c \times S^{1}$, there is a neighborhood $V$ of $y$ in $U$ such that $V \cong\left(\mathrm{cH} \times \mathbf{R}^{1}\right) \cup_{c \times \mathbf{R}^{1}}\left(\mathrm{cH} \times \mathbf{R}^{\mathrm{l}}\right)$. Since $\pi_{1}(H) \neq 0$, there is a loop in $V$ which does not bound a singular disk in $Q-Q[1]$. Hence $Q[1]$ is not 1 -LCC in $Q$.

Another interesting feature of cellular sets in polyhedra is illustrated by a similar example. Let $W$ and $H$ be as before, and let $\tilde{P}=W \cup_{H} \mathrm{cH}$, the space obtained by identifying $W$ and $\mathrm{cH}$ along the boundary $H$ of $W$ and the copy of $H$ being coned over. The polyhedron $P$ will be $\left(\tilde{P} \times S^{1}\right) \cup_{c \times S^{1}}\left(\tilde{P} \times S^{1}\right)$. If we let $\tilde{X}=\tilde{P}-$ int $B^{n+1}$, where $B^{n+1}$ is a tame $(n+1)$-cell in int $W$, then $\tilde{X}$ is a contractible subset of $\tilde{P}$ and, for each $t \in S^{1}, \tilde{X} \times t$ is cellular in the manifold $\tilde{P} \times S^{1}$. However, $X=\tilde{X} \times t$ is not cellular in $P$. If $X$ were cellular in $P$, then given a neighborhood $N$ 
of $c \times t$ which is homeomorphic to $\left(\mathrm{cH} \times \mathbf{R}^{1}\right) \cup_{c \times \mathbf{R}^{1}}\left(\mathrm{cH} \times \mathbf{R}^{1}\right)$, and a neighborhood $U$ of $X$ in $P$ such that $U \cap P[1] \subset N$, there would be a contraction $h_{t}$ of $X$ in $U$ such that, for each $x \in X, I\left(h_{t}(x), P\right)=I(x, P)$ for $t<1$ and $I(h(x), P)=1$ [9]. Suppose there were such a contraction. There are loops in $X \cap N$ arbitrarily close to $P[1]$ which do not bound in $N \cap P[n+2]$. However, all of these loops do bound in $X$. If the loop $\alpha$ in $X$ is chosen close enough to $P[1], h_{t}(\alpha) \subset N$ for $0 \leqslant t \leqslant 1$. But for some $s>0, h_{s}(D) \subset N$, where $D$ is a singular disk bounded by $\alpha$ in $X$. Hence every loop sufficiently close to $P[1]$ must bound in $N$, a contradiction.

\section{REFERENCES}

1. Ethan Aiken, Manifold phenomena in the theory of polyhedra, Trans. Amer. Math. Soc. 143 (1969), 413-473.

2. F. D. Ancel, Engulfing the tracks of a proper homotopy, notes.

3. S. Armentrout, Cellular decompositions of 3-manifolds that yield 3-manifolds, Mem. Amer. Math. Soc. No. 107 (1971).

4. J. W. Cannon, Shrinking cell-like decompositions of manifolds: codimension three, Ann. of Math. (2) 110 (1979), 83-112.

5. R. D. Edwards, Approximating certain cellular maps by homeomorphisms, manuscript.

6. R. D. Edwards and R. C. Kirby, Deformations of spaces of imbeddings, Ann. of Math. (2) 93 (1971), 63-88.

7. R. Geoghegan, Open problems in infinite-dimensional topology, Topology Proc. 4 (1979), 287-338.

8. Michael Handel, Approximating stratum preserving CE maps between CS sets of stratum preserving homeomorphisms, Geometric Topology (L. C. Glaser and T. B. Rushing, Eds.), Lecture Notes in Math., vol. 438, Springer-Verlag, New York, 1975, pp. 245-250.

9. J. Henderson, Cellularity in polyhedra, Topology Appl. 12 (1981), 267-282.

10. Approximating cellular maps between low dimensional polyhedra, Pacific J. Math. (to appear).

11. $\ldots$ A discussion of results and problems related to cellularity in polyhedra, Proc. 1980 Summer Topology Conference (Austin, Texas) (to appear).

12. R. C. Lacher, Locally flat strings and half strings, Proc. Amer. Math. Soc. 18 (1967), 299-304.

13. D. R. McMillan, Jr., A criterion for cellularity in a manifold, Ann. of Math. (2) 79 (1964), 327-337.

14. L. C. Siebenmann, Approximating cellular maps by homeomorphisms, Topology 11 (1972), 271-294.

15. Deformations of homeomorphisms on stratified sets, Comment. Math. Helv. 74 (1972), $123-163$.

16. J. R. Stallings, The piecewise-linear structure of Euclidean space, Proc. Cambridge Philos. Soc. 58 (1962), 481-488.

Department of Mathematics, University of Tennessee, KNoxville, Tennessee 37916

Current address: Department of Mathematics, Texas A \& M University, College Station, Texas 77843 